\title{
artigo
}

\section{Transtornos Mentais como Fator de Risco para Suicídio e Ideação Suicida}

\author{
Mental Disorders as a Risk Factor for Suicide and Suicidal Ideation \\ Trastornos Mentales como Factor de Riesgo de Suicidio e Ideación Suicida
}

\begin{abstract}
RESUMO
Objetivo: 0 presente trabalho tem por objetivo estudar os transtornos mentais como fatores de risco de suicídio e ideação suicida, a fim de obter informações que possam servir de ferramentas para um trabalho preventivo e tornar mais eficazes os tratamentos desses pacientes. Método: 0 método escolhido para o estudo é o de revisão bibliográfica de literatura nacional, realizada através das bases de dados SciELO, Lilacs e Medline, tendo sido selecionados artigos com os operadores booleanos: suicídio, tentativas de suicídio, ideação suicida, transtornos mentais e psicopatologia, totalizando 15 artigos para análise. Resultados: Como resultado, da análise de dados coletados, os sujeitos diagnosticados com transtorno mental apresentam maiores comprometimentos na saúde mental, aumentando a probabilidade de riscos de tentativas, ideações e suicídio. As principais comorbidades identificadas foram: depressão, ansiedade, transtornos de humor, esquizofrenia e transtornos por uso de substâncias psicoativas. Conclusão: Os transtornos mentais aumentam a probabilidade de tentativas e ideações suicidas.

DESCRITORES: Suicídio, fatores de risco, transtornos mentais.
\end{abstract}

\section{ABSTRACT}

Objective: This work aims to study mental disorders as risk factors for suicide and suicidal ideation, in order to obtain information that can serve as tools for preventive work and make the treatments of these patients more effective. Method: The method chosen for the study is the literature review of the national literature, carried out through the SciELO, Lilacs and MedLine databases, and articles were selected with boolean operators: suicide, suidide attempts, suicidal ideation, mental disorders and psychopathology, totaling 15 articles for analysis. Results: As a result, from the analysis of collected data, subjects diagnosed with mental disorders have greater impairments in mental health, increasing the probability of risk of attempts, ideations and suicide. The main associated comorbidities identified were: depression, anxiety, mood disorders due to psychoactive substance use. Conclusion: It was concluded that mental disorders increase the likelihood of suicide attempts and ideations.

DESCRIPTORS: Suicide, risk factors, mental disorders.

\section{RESUMEN}

Objetivo: Este trabajo tiene por objetivo estudiar los trastornos mentales como factores de riesgo para el suicidio y la ideación suicida, con el fin de obtener información que pueda servir como herramientas para el trabajo preventivo y hacer que los tratamientos de estos pacientes sean más eficaces. Método: El método elegido para el estudio es la revisión literaria de la lileratura nacional, realizada a través de las bases de datos SciELO, Lilacs y MedLine, y se seleccionaron artículos con operadores booleanos: suicidio, intentos de suicidio, ideación suicida, trastornos mentales y psicopatología, totalizando 15 artículos para el análisis. Resultados: Como resultados, a partir del análisis de los datos reconpilados, las personas diagnosticadas con trastornos mentales tienen mayores discapacidades em la salud mental, lo que aumenta la probabilidad de riesgo de intentos, ideaciones y suicidio. Las principales comorbilidades asociadas identificadas fueron: depresión, ansiedad, trastornos del estado de ánimo, esquizofreniay trastornos debidos al uso de sustancias psicoactivas. Conclusión: Se concluyó que los trastornos mentales aumentan la probabilidad de intentos de suicidio e ideaciones.

DESCRIPTORES: Suicidio, factores de riesgo, trastornos mentales.

RECEBIDO EM: 29/03/2021 APROVADO EM: 06/04/2021

\section{Helena Ferreira Ramos Silva}

Graduanda do Curso de Psicologia pela Universidade Santo Amaro (UNISA).

ORCID: 0000-0002-6730-8312 


\section{Silvia Helena Modenesi Pucci}

PhD Health Promotion, Docente e Coordenadora de curso de Psicologia da Universidade Santo Amaro (UNISA). ORCID: 0000-0003-2258-007X

\section{INTRODUÇÃO}

A World Health Organization (WHO $)^{1}$ entende o suicídio como o ato de matar-se deliberadamente. Há uma diversidade de atitudes e condutas que incluem o pensar em cometer o suicídio e que podem ser entendidos como comportamento de risco. Também pode-se incluir na ideação suicida: planejar, tentar e cometer o suicídio; e compreende-se como riscos os transtornos mentais, fatores sociais, psicológicos e estressores individuais de um sujeito. Estima-se ${ }^{1}$ que pacientes que cometem suicídio apresentam transtorno psiquiátrico diagnosticável em cerca de $90 \%$ dos casos; sendo, portanto, os problemas de saúde mental os preditores mais evidentes das tentativas suicidas, embora possa ser possível identificar outras causas que se manifestem momentaneamente.

Pesquisas que abordam essa temática apontam como os transtornos mentais mais evidenciados na literatura, sendo fatores desencadeadores desse ato, a depressão em consequência de seus sintomas como a desesperança, desamparo e apatia; a ansiedade em virtude de sintomas de irritabilidade, inquietação, fatigabilidade e dificuldade de concentração; transtornos de humor devido aos traços de instabilidade, agressividade e impulsividade; e a esquizofrenia e transtorno por uso de substâncias psicoativas que também são considerados preditores para o suicídio devido ao alto sofrimento psíquico e dificuldades com adversidades da vida pessoal. Outros indicadores de risco também são identificados como contribuintes para o suicídio, sendo eles fatores sociais, econômicos, demográficos e traumas da vida do sujeito ${ }^{1,2}$.

Autores afirmam que existe um elo entre o comportamento suicida e a doença ${ }^{3}$. Ou seja, a presença de um transtorno mental aumenta a vulnerabilidade e está presente na quase totalidade dos casos; mas não se pode afirmar que todo suicídio está relacionado a um transtorno mental, assim como, nem toda pessoa acometida por uma doença mental vá cometer o suicídio de fato.

Não é somente o diagnóstico de alguma psicopatologia, ou um fator, que determina a causa de uma tentativa de suicídio; mas sim, o conjunto de fatores que totaliza um processo na vida do sujeito e pode resultar no suicídio, na maioria das vezes como fuga dos problemas enfrentados no cotidiano ${ }^{3}$. A compreensão desse fenômeno pode favorecer os estudos de pesquisas para entender o suicídio e sua relação com a psicopatologia, assim como pode fornecer subsídios para a prevenção dessa problemática.

O objetivo do presente trabalho é, portanto, estudar os transtornos mentais como fatores de risco de suicídio e ideação suicida, a fim de obter informações que possam servir de ferramentas para um trabalho preventivo e tornar mais eficazes os tratamentos desses pacientes.

\section{MÉTOdOS}

$\mathrm{O}$ método de pesquisa utilizado foi a revisão bibliográfica, que se constitui de leitura de artigos e livros já publicados, apresentando vantagens para o pesquisador por possuir uma maior abrangência dos fenômenos que deseja estudar, sem, contudo, encontrar muitas restrições que com frequência ocorrem em outros tipos de pesquisa ${ }^{3}$.

Para as fontes de informações da pesquisa foram utilizadas as bases de dados SciELO, Lilacs e Medline com artigos nacionais sobre o tema, escritos na língua portuguesa e que estão dentro do período designado dos últimos 6 anos, 2015 a 2020, (realizada a pesquisa no mês de Dezembro de 2020). Foram considerados operadores booleanos: "suicídio, tentativas de suicídio, ideação suicida, transtornos mentais e psicopatologia”.

Em um primeiro momento, ocorreu a leitura dos títulos dos artigos selecionados através das palavras-chave consideradas, e foram encontrados 15 artigos para a base de dados SciELO, 82 para a base de dados Lilacs e 23 para Medline. Além dos descritores, foram necessários utilizar como critérios de inclusão para a seleção dos artigos: materiais que continham informações no resumo que indicavam uma reflexão existente entre suicídio e transtorno mental, assim como o desenvolvimento dos transtornos mentais e outros fatores de risco ao suicídio, correspondendo ao objetivo da pesquisa, o artigo estar em língua portuguesa, ser nacional e ter como resultado/conclusão resposta para o objetivo do presente trabalho. Como critérios de exclusão, foram descartados artigos que não correspondiam com o tema e objetivo do presente trabalho, assim como artigos repetidos, fora do intervalo de tempo e fora do âmbito nacional. A primeira etapa de seleção dos artigos, foi dada através da leitura dos títulos. Como segunda etapa, a leitura dos resumos, no qual foram encontrados 5 artigos para a base de dados SciELO, 7 para a base de dados Lilacs e 3 para Medline. Segue fluxograma das etapas de seleção dos artigos:

\section{RESULTADOS}

Como fonte de informação para a pesquisa, foi utilizada a base de dados SciELO, como uma das principais fontes de dados na área da saúde. Também foram utilizadas nesta pesquisa as bases de dados Lilacs e MedLine para maior abrangência dos fenômenos. Na primeira etapa houve 120 artigos das bases de dados e após os critérios de exclusão e inclusão foram selecionados 15 artigos no total, que possuíam os objetivos do tema estudado. Os demais artigos foram excluídos por não conter relação com a proposta da pesquisa.

Os artigos selecionados são dos últimos 6 anos (2015 a 2020), tendo sido evidenciado um maior número de publicações em 2020. 


\section{artigo}

Após a análise dos dados coletados, os resultados indicam que os sujeitos

diagnosticados com transtorno mental apresentam maiores comprometimentos

Figura 1. Fluxograma das etapas de seleção dos artigos.

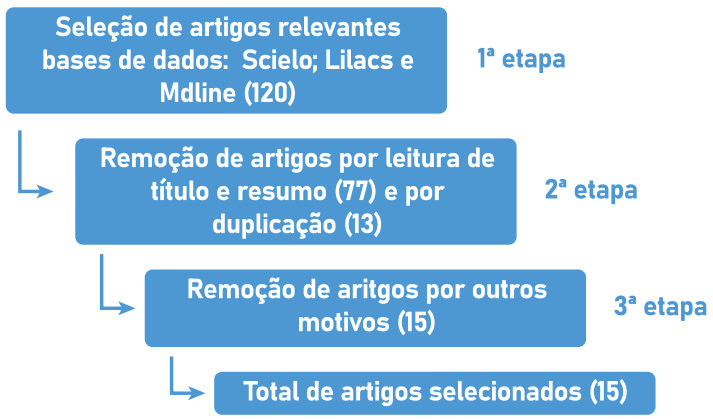

Fonte: autoria própria na saúde mental, aumentando a probabilidade de riscos de tentativas, ideações e suicídio. Identificou-se como principais comorbidades associadas: a depressão, ansiedade, transtornos de humor, esquizofrenia e transtornos por uso de substâncias psicoativas.

\section{DISCUSSÂO}

Para a realização da presente revisão bibliográfica foram selecionados 15 artigos que envolviam o tema suicídio e transtorno mental, estudos referentes ao tema devido.

Segundo Moreira et $\mathrm{al}^{4}$, verificou-se que o diagnóstico de transtorno men-

Quadro 1: Demonstrativo dos artigos elegíveis no estudo.

\begin{tabular}{|c|c|c|c|c|c|}
\hline & $\begin{array}{l}\text { AUTO- } \\
\text { RES }\end{array}$ & TÍTULO & OBJETIVO & $\begin{array}{l}\text { MÉTODO / } \\
\text { AMOSTRA }\end{array}$ & RESULTADOS \\
\hline 2020 & $\begin{array}{l}\text { Moreira } \\
\text { et al }\end{array}$ & $\begin{array}{l}\text { Transtorno Mental e } \\
\text { Risco de Suicídio em } \\
\text { Usuários de Substân- } \\
\text { cias Psicoativas: Uma } \\
\text { Revisão Integrativa. }\end{array}$ & $\begin{array}{l}\text { Analisar as evidências } \\
\text { cientificas acerca da } \\
\text { presença de transtornos } \\
\text { mentais e o risco de } \\
\text { suicídio em usuários de } \\
\text { substância psicoativas. }\end{array}$ & $\begin{array}{l}\text { Revisão integrati- } \\
\text { va da literatura. }\end{array}$ & $\begin{array}{l}\text { Usuários de substâncias psicoativas } \\
\text { apresentam maior comprometimento na } \\
\text { saúde mental aumentando a probabili- } \\
\text { dade para comorbidades psiquiátricas } \\
\text { associadas (como a depressão, esquizo- } \\
\text { frenia transtorno bipolar e ansiedade). }\end{array}$ \\
\hline 2020 & $\begin{array}{l}\text { Capis- } \\
\text { trano }\end{array}$ & $\begin{array}{l}\text { Fatores Associados à } \\
\text { Tentativa de Suicídio } \\
\text { por Pessoas com } \\
\text { Transtorno Mental. }\end{array}$ & $\begin{array}{l}\text { Identificar os fatores } \\
\text { associados à tentativa de } \\
\text { suicídio por pessoas com } \\
\text { transtorno mental. }\end{array}$ & $\begin{array}{l}\text { Estudo } \\
\text { observacional } \\
\text { e transversal } \\
\text { realizado com } \\
300 \text { pessoas com } \\
\text { transtorno mental. }\end{array}$ & $\begin{array}{c}\text { Verificou-se que ser do sexo feminino e } \\
\text { perceber a saúde como ruim e regular e } \\
\text { não aderir a terapêutica medicamentosa } \\
\text { foram os proeminentes fatores } \\
\text { associados ao histórico de tentativa de } \\
\text { suicídio. }\end{array}$ \\
\hline 2020 & $\begin{array}{l}\text { Silva et } \\
\text { al. }\end{array}$ & $\begin{array}{l}\text { A Relação entre } \\
\text { a Infecção por } \\
\text { Coronavírus e } \\
\text { Susceptibilidade a } \\
\text { Transtornos Mentais } \\
\text { e o Risco de Suicídio: } \\
\text { O que a Literatura } \\
\text { tem evidenciado? }\end{array}$ & $\begin{array}{l}\text { Identificar as evidências } \\
\text { cientificas sobre perfil } \\
\text { relacionado à saúde } \\
\text { mental em pessoas após } \\
\text { a aquisição da infecção } \\
\text { pelo coronavírus. }\end{array}$ & $\begin{array}{l}\text { Revisão } \\
\text { integrativa da } \\
\text { literatura. }\end{array}$ & $\begin{array}{l}\text { Verificou-se relação direta entre o } \\
\text { desenvolvimento de transtorno mental } \\
\text { em pacientes da COVID-19 e risco de } \\
\text { suicídio. }\end{array}$ \\
\hline 2020 & $\begin{array}{l}\text { Grigo- } \\
\text { letto } \\
\text { et al }\end{array}$ & $\begin{array}{l}\text { Tentativas de Suicídio } \\
\text { Notificadas em um } \\
\text { Hospital de Ensino no } \\
\text { Estado do Rio Grande } \\
\text { do Sul, 2014-2016. }\end{array}$ & $\begin{array}{l}\text { Caracterizar as tentativas } \\
\text { de suicídio notificadas em } \\
\text { um hospital de ensino no } \\
\text { estado do Rio Grande do } \\
\text { Sul, Brasil. }\end{array}$ & $\begin{array}{l}\text { Pesquisa } \\
\text { quantitativa e } \\
\text { retrospectiva. }\end{array}$ & $\begin{array}{l}\text { Para } 93 \% \text { das pessoas que tentaram } \\
\text { suicídio, registrou-se alguma deficiência } \\
\text { ou transtorno associado. }\end{array}$ \\
\hline 2020 & Orellana & $\begin{array}{l}\text { Transtornos Mentais } \\
\text { em Adolescentes, } \\
\text { Jovens e Adultos do } \\
\text { Consórcio de Coortes } \\
\text { de Nascimento } \\
\text { Brasileiras RPS } \\
\text { (Ribeirão Preto, } \\
\text { Pelotas e São Luís). }\end{array}$ & $\begin{array}{l}\text { Avaliar a prevalência de } \\
\text { transtornos mentais em } \\
\text { adolescentes, jovens e } \\
\text { adultos e sua relação } \\
\text { com características } \\
\text { sociodemográficas em } \\
\text { cinco estados. }\end{array}$ & $\begin{array}{l}\text { Pesquisa baseada } \\
\text { em dados coleta- } \\
\text { dos ao nasci- } \\
\text { mento de três } \\
\text { cidades brasilei- } \\
\text { ras geográficas e } \\
\text { socioeconomica- } \\
\text { mente distintas. }\end{array}$ & $\begin{array}{l}\text { Verificou-se relação entre transtorno } \\
\text { mental e risco de suicídio. }\end{array}$ \\
\hline
\end{tabular}




\begin{tabular}{|c|c|c|c|c|c|}
\hline 2020 & Neto et al & $\begin{array}{c}\text { Tentativa de } \\
\text { Suicídio, Transtorno } \\
\text { de Estresse Pós- } \\
\text { Traumático e Fatores } \\
\text { Associados em } \\
\text { Mulheres do Recife. }\end{array}$ & $\begin{array}{l}\text { Investigar a associação da } \\
\text { tentativa de suicídio com } \\
\text { o transtorno de estresse } \\
\text { pós-traumático. }\end{array}$ & $\begin{array}{c}\text { Estudo } \\
\text { transversal } \\
\text { aninhado em um } \\
\text { estudo de corte } \\
\text { prospectivo com } \\
644 \text { mulheres. }\end{array}$ & $\begin{array}{c}\text { Verificou-se relação entre tentativa } \\
\text { de suicídio e transtorno de estresse } \\
\text { pós-traumático. }\end{array}$ \\
\hline 2020 & $\begin{array}{c}\text { Oliveira } \\
\text { et al }\end{array}$ & $\begin{array}{c}\text { Características } \\
\text { das Tentativas de } \\
\text { Suicídio Atendidas } \\
\text { pelo Serviço de } \\
\text { Emergência Pré- } \\
\text { Hospitalar: Um Estudo } \\
\text { Epidemiológico de } \\
\text { Corte Transversal. }\end{array}$ & $\begin{array}{l}\text { Descrever o perfil das } \\
\text { vítimas de tentativas de } \\
\text { suicídio atendidas pelo } \\
\text { Corpo de Bombeiros } \\
\text { de um município do } \\
\text { semiárido brasileiro. }\end{array}$ & $\begin{array}{c}\text { Estudo } \\
\text { transversal } \\
\text { com vítimas } \\
\text { de tentativa de } \\
\text { suicídio atendidas } \\
\text { por bombeiros. }\end{array}$ & $\begin{array}{l}\text { Verificou-se maior presença de } \\
\text { transtorno mental entre os homens } \\
\text { atendidos pelos bombeiros, que } \\
\text { tentaram suicídio. }\end{array}$ \\
\hline 2019 & $\begin{array}{l}\text { Nasci- } \\
\text { mento }\end{array}$ & $\begin{array}{l}\text { Associação entre } \\
\text { Transtornos } \\
\text { Alimentares, Suicídio e } \\
\text { Sintomas Depressivos } \\
\text { em Universitários de } \\
\text { Cursos de Saúde. }\end{array}$ & $\begin{array}{l}\text { Identificar sintomas de } \\
\text { transtornos alimentares } \\
\text { e possíveis associações } \\
\text { com o risco d suicídio e } \\
\text { sintomas depressivos. }\end{array}$ & $\begin{array}{l}\text { Estudo de corte } \\
\text { transversal, } \\
\text { realizado com } \\
271 \text { estudantes. }\end{array}$ & $\begin{array}{l}\text { Verificou-se correlação entre } \\
\text { transtornos alimentares e depressão } \\
\text { com risco de suicídio. }\end{array}$ \\
\hline 2019 & $\begin{array}{l}\text { Minayo; } \\
\text { Figueiredo; } \\
\text { Mangas. }\end{array}$ & $\begin{array}{c}\text { Estudos das } \\
\text { Publicações Cientificas } \\
\text { (2002 - 2017) Sobre } \\
\text { Ideação Suicida, } \\
\text { Tentativas de Suicídio } \\
\text { e Autonegligência de } \\
\text { Idosos Internados em } \\
\text { Instituições de Longa } \\
\text { Permanência. }\end{array}$ & $\begin{array}{l}\text { Apresentar publicações } \\
\text { sobre ideação suicida, } \\
\text { tentativa de suicídio } \\
\text { e autonegligência em } \\
\text { idosos internados em ILPI. }\end{array}$ & $\begin{array}{l}\text { Estudo baseado } \\
\text { em trabalhos } \\
\text { científicos. }\end{array}$ & $\begin{array}{l}\text { Verificou-se que a depressão entre } \\
\text { outros fatores pode levar os idosos ao } \\
\text { comportamento suicida. }\end{array}$ \\
\hline 2018 & Botti & $\begin{array}{l}\text { Características e } \\
\text { Fatores de Risco do } \\
\text { Comportamento } \\
\text { Suicida entre } \\
\text { Homens e Mulheres } \\
\text { com Transtornos } \\
\text { Psiquiátricos. }\end{array}$ & $\begin{array}{l}\text { Identificar características } \\
\text { do comportamento } \\
\text { suicida e fatores de risco } \\
\text { de homens e mulheres } \\
\text { com transtornos } \\
\text { psiquiátricos. }\end{array}$ & $\begin{array}{l}\text { Estudo } \\
\text { quantitativo } \\
\text { realizado com } \\
410 \text { prontuários } \\
\text { de pacientes } \\
\text { psiquiátricos. }\end{array}$ & $\begin{array}{l}\text { Evidenciaram-se particularidades } \\
\text { do comportamento suicida } \\
\text { entre pacientes psiquiátricos em } \\
\text { tratamento. }\end{array}$ \\
\hline 2017 & Borba et al & $\begin{array}{l}\text { Perfil do Portador de } \\
\text { Transtorno Mental } \\
\text { em Tratamento no } \\
\text { Centro de Atenção } \\
\text { Psicossocial (CAPS). }\end{array}$ & $\begin{array}{l}\text { Caracterizar o perfil do } \\
\text { portador de transtorno } \\
\text { mental em tratamento } \\
\text { no Centro de Atenção } \\
\text { Psicossocial. }\end{array}$ & $\begin{array}{l}\text { Estudo descritivo } \\
\text { realizado com } \\
300 \text { portadores } \\
\text { de transtorno } \\
\text { mental. }\end{array}$ & $\begin{array}{l}33,6 \% \text { possuíam transtorno afetivo } \\
\text { bipolar, } 19,1 \% \text { esquizofrenia e } 18,6 \% \\
\text { depressão. } 78,6 \% \text { necessitaram de } \\
\text { internação e } 67,7 \% \text { tentaram suicídio. }\end{array}$ \\
\hline 2016 & $\begin{array}{l}\text { Fer- } \\
\text { nandes; } \\
\text { Ferreira; } \\
\text { Castro. }\end{array}$ & $\begin{array}{l}\text { Perfil Epidemiológico } \\
\text { das Tentativas de } \\
\text { Suicídio em Palmas- } \\
\text { Tocantins, de } 2010 \text { a } \\
2014 .\end{array}$ & $\begin{array}{c}\text { Descrever o perfil } \\
\text { epidemiológico das } \\
\text { tentativas de suicídio } \\
\text { notificadas em residentes } \\
\text { de Palmas. }\end{array}$ & $\begin{array}{l}\text { Estudo } \\
\text { epidemiológico } \\
\text { descritivo/ } \\
\text { quantitativo. }\end{array}$ & $\begin{array}{l}\text { Identificou-se diferentes tipos de } \\
\text { deficiência/transtorno mental em } \\
23,47 \% \text { nas tentativas de suicídio. }\end{array}$ \\
\hline 2015 & Pires et al. & $\begin{array}{c}\text { Indicadores de } \\
\text { Risco para Tentativa } \\
\text { de Suicídio por } \\
\text { Envenenamento: Um } \\
\text { Estudo Caso Controle. }\end{array}$ & $\begin{array}{l}\text { Investigar possíveis } \\
\text { indicadores de risco de } \\
\text { tentativas de suicídio por } \\
\text { envenenamento. }\end{array}$ & $\begin{array}{l}\text { Estudo de caso } \\
\text { controle com } 220 \\
\text { pessoas. }\end{array}$ & $\begin{array}{l}\text { Foram considerados indicadores } \\
\text { de risco para a tentativa de suicídio } \\
\text { estar dependente financeiramente de } \\
\text { terceiros, abuso sexual na infância, } \\
\text { ideação suicida e histórico de } \\
\text { transtorno mental na família. }\end{array}$ \\
\hline
\end{tabular}




\begin{tabular}{|c|c|c|c|c|c|}
\hline 2015 & $\begin{array}{l}\text { Vasconce- } \\
\text { los; Lôbo; } \\
\text { Neto. }\end{array}$ & $\begin{array}{l}\text { Risco de Suicídio } \\
\text { e Comorbidades } \\
\text { Psiquiátricas } \\
\text { no Transtorno } \\
\text { de Ansiedade } \\
\text { Generalizada. }\end{array}$ & $\begin{array}{l}\text { Investigar o risco de } \\
\text { suicídio nos pacientes } \\
\text { com transtorno de } \\
\text { ansiedade generalizada. }\end{array}$ & $\begin{array}{c}\text { Estudo } \\
\text { transversal com } \\
253 \text { pacientes. }\end{array}$ & $\begin{array}{c}\text { Verificou-se comorbidade psiquiátrica } \\
\text { em } 88,1 \% \text { dos entrevistados. A mais } \\
\text { prevalente foi depressão maior } \\
(53,7 \%) \text {, seguida de distimia }(21,4 \%) \text { e } \\
\text { depressão recorrente }(14,6 \%) \text {. } 0 \text { risco } \\
\text { de suicídio foi observado em } 54,8 \% \\
\text { maior dos indivíduos com TAG. }\end{array}$ \\
\hline
\end{tabular}

tal com uso de substâncias psicoativas apresenta maior comprometimento na saúde mental do sujeito, elevando a probabilidade de maiores comorbidades, como a depressão, esquizofrenia, transtorno bipolar e ansiedade, aumentando consideravelmente o risco de suicídio em até 5,7 vezes. Corroborando com este resultado, um estudo realizado no Brasil, por Hess et $\mathrm{al}^{5}$, com 94 homens identificou maior ocorrência de transtornos psiquiátricos por indivíduos usuários de substâncias psicoativas, provocando maior risco de suicídio, principalmente ao que se refere no consumo de múltiplas substâncias.

No segundo artigo referenciado por Capistrano et $\mathrm{al}^{6}$, compreendeu-se que quando o sujeito com histórico de transtorno mental não adere a terapêutica medicamentosa proposta, identifica-se a piora do seu quadro clínico, favorecendo o desenvolvimento de fatores associados as tentativas de suicídio $(67,7 \%)$ com pacientes em tratamento em um CAPS de Curitiba, sendo $47,3 \%$ mulheres e $20,3 \%$ homens. Dados de estudo realizado com a população canadense identificou que a prevalência de tentativas de suicídio entre pessoas com diagnóstico de esquizofrenia foi de $32,2 \%$, enquanto a população sem o diagnóstico da doença 2,8\%, demonstrando que aqueles com esquizofrenia apresentam 6 vezes mais exequibilidade de tentar suicídio 7 .

Uma pesquisa realizada por Santos et $\mathrm{al}^{8}$ durante a rápida e repentina proliferação do vírus da COVID-19, evidenciou que um dos impactos provocados pelo coronavírus está diretamente relacionado à saúde mental da população, ocasionando uma alta prevalência de sofrimentos psicológicos, psiquiátricos e dos sintomas de ansiedade, depressão e transtornos de estresse pós-traumático, aumentando as chances de risco de suicídio. De encontro a este resultado, um trabalho ${ }^{9}$ verificou que a grande maioria dos pacientes que contraíram o coronavírus pode enfrentar problemas psicológicos desencadeados pelo cenário da pandemia, incluindo depressão, ansiedade e estresse, e pacientes psiquiátricos diagnosticados com depressão ou esquizofrenia, exigem dos profissionais de saúde total atenção, pois o risco de cometer suicídio é significativamente maior do que comparado a população geral.

\section{Dados de estudo}

realizado com a

população canadense

identificou que

a prevalência de

tentativas de suicídio

entre pessoas com

diagnóstico de

esquizofrenia foi de

$32,2 \%$...
Na pesquisa realizada por Grigoletto et a ${ }^{10} \mathrm{em}$ um hospital de ensino no estado do Rio Grande do Sul, Brasil, caracterizou as tentativas de suicídio registradas entre os anos de 2014 e 2016, uma totalidade dos pacientes que possuíam qualquer tipo de transtorno associado a tentativas de suicídio. Identificou-se notificações com transtornos mentais e comportamentais sendo 93\% dos pacientes que tentaram suicídio, apresentavam alguma comorbidade ou transtorno associado, principalmente a depressão. Este achado também foi verificado em outro trabalho ${ }^{11}$ que comparou a elevada frequência de comorbidades psicológicas, como a depressão maior, ansiedade generalizada e fobia social relacionadas ao risco de suicídio. De encontro com esses resultados, um estudo finlandês, ao analisar a prevalência e comorbidade de transtornos mentais (93\%) em uma amostra aleatória de 229 vítimas de suicídio, verificou entre os transtornos mais prevalentes a depressão (59\%) e dependência ou abuso de álcool $(43 \%)^{12}$.

Segundo Neto et $\mathrm{a}^{13} \mathrm{em}$ pesquisa realizada na cidade de Recife com $644 \mathrm{mu}-$ lheres entre 18 a 49 anos, cadastradas na Estratégia Saúde da Família, verificou que as tentativas de suicídio além de estarem relacionadas ao transtorno de estresse pós-traumático e violência pelo parceiro íntimo, também pode ser resultado de fatores sociais, econômicos e demográficos. A prevalência e chances de tentativas suicidas nas mulheres que apresentavam transtorno de estresse pós-traumático foi aproximadamente 5 vezes maior das que não apresentavam nenhum sintoma. Corroborando com esses resultados, na Irlanda do Norte, um estudo detectou que o risco de ideação, planos e tentativas de suicídio são substancialmente maiores em pessoas com 
transtornos mentais. As taxas de transtorno de estresse pós-traumático são as mais elevadas no país, com 4,3\% em mulheres e $2,3 \%$ nos homens ${ }^{14}$.

Um estudo transversal ${ }^{15}$ contendo dados do atendimento a vítimas de tentativas de suicídio, realizado por bombeiros militares do estado de Alagoas, observou que a presença de um transtorno mental foi predominantemente entre o sexo masculino com $76,5 \%$. No sexo feminino observou-se predomínio de intoxicação. De encontro a esse resultado, outro estudo com 164 casos de sujeitos que tentaram suicídio por intoxicações exógenas, $70,1 \%$ eram mulheres e $29,1 \%$ homens $^{16}$, assim como em um estudo realizado por Corradi et al na cidade de São Paulo, que identifica como principal meio nas tentativas de suicídio de adultos a intoxicação e envenenamento ${ }^{17}$.

$\mathrm{O}$ artigo de Nascimento ${ }^{18}$, verificou em um estudo transversal realizado com 271 universitários de cursos de saúde, possíveis associações de sintomas de transtornos alimentares e depressivos com o risco de suicídio. Nesse mesmo estudo identificou-se que os estudantes que apresentaram sintomas de anorexia nervosa e bulimia nervosa, assim como aqueles com sintomatologia de depressão possuíam maior probabilidade de risco para cometer suicídio. Os principais subtipos de transtornos alimentares evidenciados na literatura é a anorexia e bulimia nervosa, em uma revisão integrativa de literatura evidenciou-se segundo Veras et a ${ }^{19}$ correlação positiva na relação dos transtornos alimentares e comportamento suicida.

Segundo Minayo et $\mathrm{al}^{20}$, em uma pesquisa realizada com idosos internados em Instituições de Longa Permanência, estes apresentaram maiores comportamentos suicidas comparados a população de idosos em geral. Na presente revisão integrativa identificou-se como riscos de ideações e tentativas de suicídio entre idosos problemas psiquiátricos, sendo a depressão o maior de todos, ansiedade, transtorno de personalidade, distúrbio comportamental, além de fragilidade e limitações de causas próprias da idade. De fato, foi verificado que problemas físicos, neurobiológicos, psicológicos e sociais são indicadores de fatores de risco na contribuição de pensamentos, sentimentos, tentativas e execução do suicídio cometido por idosos. A depressão é a enfermidade com maior relevância dentre os fatores associados ao suicídio nessa faixa etária ${ }^{21}$.

Achados de um estudo quantitativo por Botti et al ${ }^{22}$ com 410 prontuários de pacientes psiquiátricos em um Centro de Atenção Psicossocial, assegura a afirmação de que o maior número de sujeitos com ideações ou tentativas de suicídio possuem comorbidades psiquiátricas significativas. O presente estudo identificou como comportamento suicida das mulheres o transtorno de humor, enquanto os homens apresentavam transtornos devido ao uso de substâncias psicoativas. Sendo os transtornos mentais um dos principais fatores de risco para o suicídio, os resultados de um estudo realizado em um hospital psiquiátrico espanhol com 79 casos de suicídio consumado evidenciaram que $54,3 \%$ apresentaram transtorno afetivo; $37,1 \%$ transtorno depressivo; $14,3 \%$ transtorno bipolar; $17,1 \%$ transtorno psicótico e $42,9 \%$ por transtornos relacionados ao uso de substâncias. Ainda em estudo realizado com amostra espanhola, a pesquisa confirma a prevalência de uma alta taxa de transtornos psiquiátricos nos indivíduos que cometem suicídio ${ }^{23}$.

Segundo Borba et $\mathrm{al}^{24}$, em estudo descritivo realizado com 300 pacientes de dois CAPS de Curitiba diagnosticados com algum transtorno mental, verificou-se no que diz respeito á tentativas de suicídio, que indivíduos portadores de transtornos mentais possuem de 3 a 12 vezes maior risco de cometer suicídio comparados àqueles sem enfermidade psiquiátrica. As comorbidades prevalentes dos pacientes diagnosticados com transtorno mental no CAPS foi o transtorno afetivo bipolar (33,6\%), esquizofrenia $(19,1 \%)$ e depressão (18,6\%). 67,7\% dos pacientes tentaram suicídio e 78,6\% necessitaram de internação para melhor controle de seu estado psicológico. Resultados encontrados em outro estudo apontam que indivíduos
O presente estudo identificou como

comportamento

suicida das mulheres

o transtorno de

humor, enquanto

os homens

apresentavam

transtornos devido

ao uso de substâncias

psicoativas.

que sofrem de transtornos afetivos possuem maior probabilidade de suicídio ${ }^{25}$.

Uma pesquisa realizada em Palmas, Tocantins por Fernandes e Ferreira ${ }^{26}$, analisou-se as notificações de 656 casos de tentativas de suicídio, sendo as tentativas 12,15 vezes maiores do que o suicídio consumado. Na presente amostra verificou-se relação abaixo das porcentagens, se comparada as demais pesquisas, sendo $33,11 \%$ os indivíduos identificados com transtornos mentais, e a depressão, o transtorno com maior prevalência na associação ao suicídio. Em contrapartida, um estudo realizado na região da América Latina e Caribe, nota-se aumento nas taxas de suicídio em quase todos os países latino-americano, classificados como fatores de risco suicida em potencial a associação com transtorno de ansiedade e transtorno depressivo maior ${ }^{27}$. 


\section{artigo}

$\mathrm{Na}$ amostra de uma pesquisa realizada na emergência do Hospital de Restauração na cidade de Recife, verificou-se indicadores de risco de tentativa e suicídio, questões financeiras, abuso sexual na infância, ideações suicidas, apresentar comorbidades psiquiátricas e transtornos mentais (como episódio depressivo maior e ansiedade generalizada). As probabilidades de um sujeito apresentar comportamento de autodestruição na presença de quatro fatores de risco simultaneamente aumentariam as chances da tentativa de suicídio por envenenamento em até $94 \%{ }^{28}$.

Dados semelhantes foram obtidos em outro estudo por Vasconcelos et al ${ }^{29}$ evidenciando que sujeitos com transtorno de ansiedade generalizada (TAG) apresentam elevada relação com outras comorbidades psiquiátricas, nomeadamente os transtornos de humor e depressão maior.
A presença desse transtorno aumenta significativamente o risco de suicídio, sendo 54\% indivíduos com TAG. Corroborando com os resultados acima, sujeitos com TAG possuem risco elevado com outras comorbidades psiquiátricas como a depressão/distimia, além de estar associado a maiores chances de tentativas e ideações suicidas ao longo da vida ${ }^{30}$.

\section{CONCLUSÃO}

O desenvolvimento da presente pesquisa possibilitou identificar os principais transtornos mentais prevalentes como fatores de risco no comportamento e ideação ao suicídio. Entre eles depressão, ansiedade, transtornos de humor, transtorno por uso de substâncias e esquizofrenia. Os estudos analisados permitiram concluir que o desenvolvimento de uma comorbidade psicopatológica contribui como fator de risco para o suicídio. Dessa forma, os transtornos mentais são classificados como um dos principais fatores de risco para ideação, tentativa e suicídio. No entanto, é preciso entender que nem todo indivíduo diagnosticado com alguma comorbidade psiquiátrica vá se suicidar, e que nem todo suicida possui diagnóstico de transtorno mental.

Estudos sobre essa temática são de extrema importância pois oferecem informações aos profissionais de saúde e para a população, contribuindo assim para melhor conhecimento e aprofundamento sobre o assunto. Faz-se importante a elaboração de novas pesquisas contendo discussôes sobre melhores estratégias de atendimento para prevenção e cuidado de indivíduos com comportamento suicida, a fim de minimizar essa problemática. -

\section{REFERÊNCIAS}

1. Organização Mundial da Saúde; Mental Health and Substance Use. Brasil: WHO; 2020. [acesso em 01.02.2021] Disponível em https://www.who.int/teams /ment al-health-and-substanceuse/suicid e-data

2. Meneghel, SN; Moura, R. Suicídio, cultura e trabalho em município de colonização alemã no sul do Brasil. Interface (Botucatu) [online]. 2016, [acesso em 19 mar 2021] vol.22, n.67, pp.1135-1146. Disponivel em https://www.scielo.br/pdf/icse/ v22n67/1807-5762-icse-1807-576220170269.pdf

3. Botega, NJ, Crise Suicida: Avaliação e Manejo. Porto Alegre: Artmed, 2015.

4. Gil, AC. Métodos e técnicas de pesquisa social. 5. ed. São Paulo: Atlas, 1999.

5. Moreira, RMM; Oliveira EM; Lopes RE; Lopes MVO; Félix TA; Oliveira LS. Transtorno Mental e Risco de Suicídio em Usuários de Substâncias Psicoativas: Uma Revisão Integrativa. SMAD, Rev Eletrônica Saúde Mental Álcool Drog. [periódico na Internet]. 2020 jan.-fev; [acesso em 19 mar 2021]; 16(1):1-10 Disponível em: http://pepsic.bvsalud.org /pdf/smad/v16n1/v16n1a16.pdf

6. Hess ARB; Almeida RMM; Moraes AL; Comorbidades Psiquiátricas em Dependentes Químicos em Abstinência em Ambiente Protegido. Estudos de Psicologia, [acesso em 19 mar 2021] janeiro-abril/2012, 171-178. Disponivel em:https:// www.scielo.br/pdf/epsic/v17n1/21.pdf

7. Borba LO; Ferreira ACZ; Capistrano FC; Kalinke LP; Maftum GJ; Fatores Associados à Tentativa de Suicídio por Pessoas com Transtorno Mental. REME - Min Enferm. 2020. [acesso em 18 mar 2021]; 24:e-1284. Disponivel em: https://cdn.publisher.gn1. link/reme.org.br/pdf/e1284.pdf

8. Thomson EF; Hollister B; Schizophrenia and Suicide Attempts: Findings from a Representative Community - Based Canadian Sample. Hindawi Publishing Corporation Schizophrenia Research and Treatment; [acesso em 19 mar 2021]; Volume 2016, Article ID 3165243, 11 pages; Disponivel em:https://downloads. hindawi.com/journals/schizort/2016/3165243.pdf

9. Santos, VD; Candeloro, RJ. Trabalhos Acadêmicos: Uma orientação para a pesquisa e normas técnicas. Porto Alegre: AGE, Ltda, 2006.

10. Soares RJO; COVID-19 e Riscos Psicossociais: Um Alerta Sobre o Suicídio. Brazilian Journal of Health Review 2021. [acesso em 19 mar 2021] Curitiba, v.4, n.1, p 1859-1870 jan./feb. Disponivel em https://www.brazilianjour nals.com/index.php/ BJHR/article/view/23639

11. Grigoletto AP; Souto VT; Terra MG; Tisott ZL; Ferreira C. N; Tentativas de Suicídio Notificadas em um Hospital de Ensino no Estado do Rio Grande do Sul, 2014 - 2016. Revista Online de Pesquisa Cuidado é fundamental. Universidade Federal do Rio de Janeiro. Escola de Enfermagem Alfredo Pinto. 2020. [acesso em 18 mar 2021] jan/dez 12: 413-419 Disponivel em: http:// seer.unirio.br/ index.php/cuidadofundamental/article/viewFile/8349/pdf_1

12. Orellana JDY; Ribeiro MRC; Barbieri MA; Saraiva MC; Cardoso VC; Bettiol H; Silva AAM; Barros FC; Gonçalves $\mathrm{H}_{\text {; Wehrmeister }}$ FC; Menezes AMB; Del-bem CM; Horta BL; Transtornos Mentais em Adolescentes Jovens e Adultos do Consócio de Coortes de Nascimento Brasileiras RPS (Ribeirão Preto, Pelotas e São Luís). Cad. Saúde Pública [online]. 2020. [acesso em 19 mar 2021]. Vol 
36, n.2, e00154319. Disponível em: https://www.scielo.br /pdf/ csp/v36n2/1678-4464-csp-36-02-e00154319.pdf

13. Henriksson MM; Aro $\mathrm{HM}_{\text {; }}$ Marttunen MJ; Heikkinen ME; Isometsa ET; Kuoppasalmi KI; Lonnqvist JK; Mental Disorders and Comorbidity in Suicide. June 1993. [acesso em 18 mar 2021]. Am J Psychiatry 150:6. Disponivel em: https://pubmed. ncbi.nlm.nih.gov/ 8494072

14. Neto PJAV; Moreira RS; Júnior FJMO; Ludermir AB; Tentativa de Suicídio, Transtorno de Estresse Pós-Traumático e Fatores Associados em Mulheres do Recife. Rev. bras. Epidemol. [acesso em 18 mar 2021] vol.23, Rio de Janeiro, 2020. Disponível em: https://www.scielo.br/pdf/rbepid/v23/1980-5497-rbepid23-e200010.pdf

15. O'neill S; Murphy FFS; Corry C; Bolton D; Devine B; Ennis E; Bunting B; Patterns Of Suicidal Ideation and Behavior in Northern Ireland and Associations With Conflict Related Trauma. Journal Plos One [acesso em 18 mar 2021] 9(3): e 91532, mar 2014. Disponivel em: file:///C:/Users/asus /Downloads/Patterns\%20 of $\% 20$ Suicidal $\% 20$ Ideation $\% 20$ and $\% 20$ Behavior $\% 20$ in $\% 20$ Northern.pdf

16. Oliveira JWT; Magalhães APN; Barros AC; Monteiro EKR; Souza CDF; Alves VM; Características das Tentativas de Suicídio Atendidas Pelo Serviço de Emergência Pré-Hospitalar: Um Estudo Epidemiológico de Corte Transversal. J. bras. Psiquiatr. [acesso em 18 mar 2021] vol. 69 n.4 Rio de Janeiro Oct/ Dec. 2020. Disponivel em: https://www.scielo.br /pdf/jbpsiq/ v69n4/1982-0208-jbpsiq-69-04-0239.pdf

17. Vieira LP; Santana VTP; Suchara EA; Caracterização de Tentativas de Suicídios Por Substâncias Exógenas. Cad. Saúde col. [acesso em 19 mar 2021] vol 23, n.2, pp 118-123. 2015. Disponivel em: https://www.scielo.br/pdf/cadsc/ v23n2/1414462X-cadsc-23-2-118.pdf

18. Corradi L; Rodrigues CL; Oliveira JC; Tentativa de Suicídio em Residentes de São Paulo: Aspectos Epidemiológicos. Saúde Coletiva (Barueri) [acesso em 05 de abril 2021]; n.59 (10); São Paulo 2020. Disponível em: https://doi.org/10.36489/saudecoletiva.2020v10i59p4214-4223

19. Nascimento VS; Santos AV; Arruda SB; Silva GA; Cintra JDS; Pinto TCC; Ximenes RCC; Associação Entre Transtornos Alimentares, Suicídio e Sintomas Depressivos em Universitários de Cursos de Saúde. J. einstein [acesso em 18 mar 2021]. São Paul. 12 dez 2019; 1:eA04908 Disponível em: https://www.scielo.br/ pdf/eins/v18/pt_2317-6385-eins-18-eA04908.pdf

20. Veras JLA; Ximenes RCC; Vasconcelos FMN; Medeiros BF; Sougey EB; Relação Entre Comportamento Suicida e Transtornos Alimentares: Uma Revisão Sistematizada. Rev. Pesqui. Univ. Fed. Estao Rio. J., on line. [acesso em 18 mar 2021]; 10(1): 29294, jan-mar. 2018. Disponivel em: http://www.index-f.com/ pesquisa/2018pdf/101289.pdf

21. Minayo MCS; Figueiredo AEB; Mangas RMN; Estudos das Publicações Cientificas (2002-2017) Sobre Ideação Suicida, Tentativas de Suicídio e Autonegligência de Idosos Internados em Instituições de Longa Permanência. Ciênc. Saúde coletiva [acesso em 16 mar 2021] vol.24 n.4 Rio de Janeiro abr.2019. Disponivel em: https://www.scielo.br/ pdf/csc/v24n4/14138123-csc-24-04-1393.pdf

22. Minayo MCS; Cavalcante FG; Suicídio Entre Pessoas Idosas: Revisão da Literatura. Rev. Saúde Pública; [acesso em 18 mar 2021]; 44(4); 750-7. 2010. Disponivel em: https://www.scielo. br/pdf/rsp /v44n4/20.pdf

23. Botti NCL; Cantão L; Silva AC; Dias TG; Menezes LC; Castro RAS; Características e Fatores de Risco do Comportamento Suicida Entre Homens e Mulheres com Transtornos Psiquiátricos. 2018; [acesso em 18 mar 2021] Artigo Original; Disponivel em: https://docs.bvsalud.org/biblioref/2018/05/883500/54280231505-1-pb.pdf

24. Gómez-duran ES; Forti-buratti MA; Lópes BG; Ibanez AB; Fumadó CM; Transtornos Psiquiátricos En Los Casos de Suicidio Consumado En Un Área Hospitalaria Entre 2007-2010. Elsevier Rev. De Psiquiatria y Salud Mental [acesso em 18 mar 2021] vol.9. n.1. p. 31-38 2016. Disponivel em: https://www.sciencedirect.com/science/article/abs/pii/S188898911400038X?via\%3Dihub

25. Borba LO; Ferreira ACZ; Capistrano FC; Kalinke LP; Maftum GJ; Fatores Associados à Tentativa de Suicídio por Pessoas com Transtorno Mental. REME - Ver. Min Enferm. [acesso em 17 mar 2021] 2020. Disponível em: https://cdn.publisher.gn1.link/reme. org.br/pdf/e1284.pdf

26. Fernandes FN; Scippa AM; Comportamento Suicida no Transtorno Afetivo Bipolar e Características Sociodemográficas, Clínicas e Neuroanatômicas Associadas. 2012. Disponível em: https://www.scielo.br/ pdf/rpc/v40n6/v40n6a03.pdf

27. Fernandes DAA; Ferreira NS; Castro JGD; Perfil Epidemiológico das Tentativas de Suicídio em Palmas - Tocantins, de 2010 a 2014. 2016 Disponivel em: https://www.tempusactas .unb.br/ index.php/tempus/article/view/2016/1706

28. Teti GL; Rebok F; Rojas SM; Grendas L; Darayf. M; Systematic Review Of Risk Factors For Suicide and Suicide Attempt Among Psychiatric Patients In Latin America and Caribbean. 2014. Disponivel em: https://www.scielosp .org/pdf/rpsp/2014. v36n2/124-133

29. Pires MCC; Raposo MCF; Sougey EB; Filho OCB; Silva TS; Passos MP; Indicadores de Risco Para Tentativa de Suicídio por Envenenamento: Um Estudo Caso-Controle. 2015. Disponível em: https://www.scielo.br/pdf/jbpsiq/v64n3/0047-2085-jbpsiq-64-3-0193.pdf

30. Vasconcelos JRO; Lôbo ACS; Neto VLM; Risco de Suicídio e Comorbidades Psiquiátricas no Transtorno de Ansiedade Generalizada. 2015. Disponível em: https://www.scielo.br/pdf/jbpsiq/ v64n4/0047-2085-jbpsiq-64-4-0259.pdf

31. Thibodeau MA; Welch PG; Sareen J; Anxiety Disorders Are Independently Associated With Suicide Ideation And Attempts: Propensity Score Matching In Two Epidemiological Samples. 2013. Disponível em: https://pubmed.ncbi.nlm. nih. gov/24108489 